\title{
Borylative coupling
}

Chem. Sci. http://doi.org/dbtw (2019).

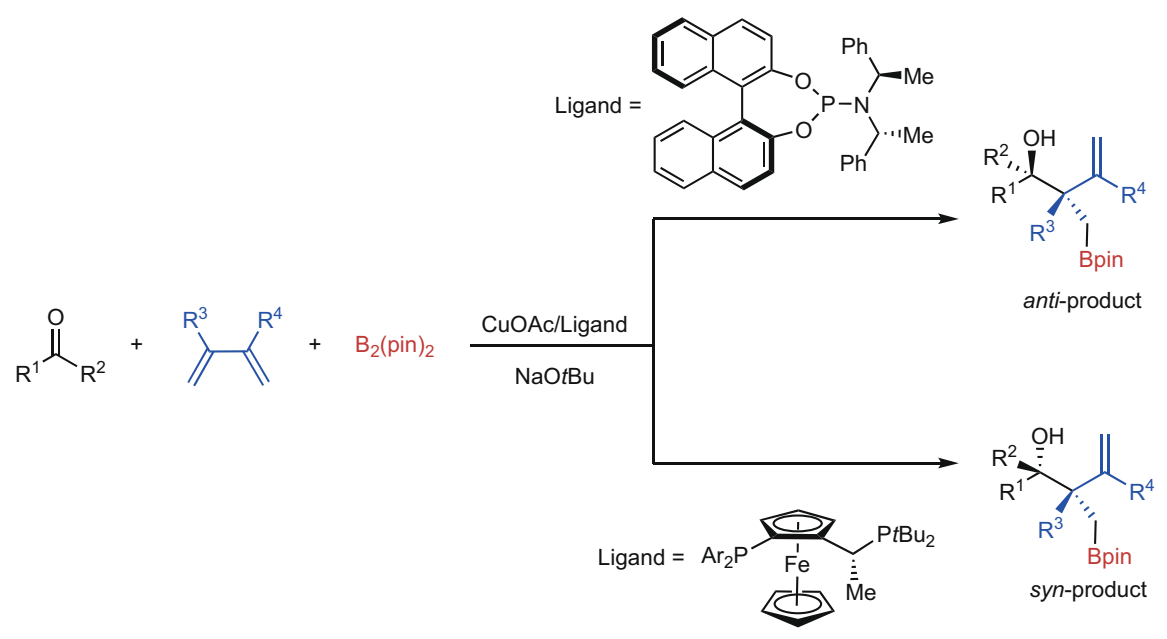

Homoallylic alcohols have long been prepared by the allylation of carbonyls. Allyl silanes are perhaps the best-known nucleophiles used (Hosomi-Sakurai reaction), but boron reagents and others have also been successfully employed. Some challenges still remain: tertiary homoallylic alcohols require additions to ketones (more difficult from both a selectivity and reactivity point-of-view) and also, when dealing with substituted allyl nucleophiles, the issue of regio- and diastereoselectivity complicates matters. Now Martin Oestreich and colleagues at Technische Universität Berlin have developed a copper-catalysed coupling of dienes, ketones and diborons, giving tertiary homoallylic alcohols with high and switchable enantio-, regio- and diastereoselectivity.

Importantly, the selectivity of the process was controlled by the ligand. Using phosphoramidte ligands led to the formation of anti-products as the major diastereomer, whereas Josiphos gave the syn-product. This observation was found to be general across a range of substrates, and yields and selectivities were normally very good. The best results were obtained with aryl alkyl ketones, though alkyl-alkyl and enones also worked. The authors propose a mechanism occurring firstly via the addition of $\mathrm{Cu}$-Bpin to the diene, forming an allylcopper nucleophile. This adds to the ketone through a six-membered transition state in a step that determines the enantiomeric and diastereomeric ratios.

One of the most interesting parts of this study is the synthetic utility. The products are highly functionalized, and as can be imagined the authors could demonstrate significant potential as building blocks for other functionalized chiral molecules. The alkyl borane could be used for SuzukiMiyaura couplings. Alternatively, oxidative work-up breaks the $\mathrm{C}-\mathrm{B}$ bond to give the diol. Beyond these relatively straightforward procedures, the authors also showed a number of other derivatization routes involving reactivity at the double bond and the $\mathrm{OH}$ groups, without any meaningful erosion of enantiomeric excess.

\section{Enda Bergin}

Published online: 11 October 2019

https://doi.org/10.1038/s41929-019-0373-9 